Published in final edited form as:

Leuk Lymphoma. 2013 August ; 54(8): 1836-1839. doi:10.3109/10428194.2013.796055.

\title{
MicroRNAs and B cell receptor signaling in chronic lymphocytic leukemia
}

\author{
Marek Mraz ${ }^{1,2}$ and Thomas J. Kipps ${ }^{1}$ \\ ${ }^{1}$ Rebecca and John Moores Cancer Center, University of California San Diego, La Jolla, CA, USA \\ ${ }^{2}$ Central European Institute of Technology, Masaryk University, Brno, Czech Republic
}

\begin{abstract}
The relative expression levels of certain microRNAs (miRNAs) correlate with known prognostic markers in chronic lymphocytic leukemia (CLL), such as leukemia-cell expression of zetaassociated protein of $70 \mathrm{kDa}$ (ZAP-70), use of unmutated immunoglobulin heavy-chain variable region genes (IGHV), chromosomal abnormalities or dysfunctional p53. Here we review studies that provide evidence suggesting that certain miRNAs (e.g. miR-155, miR-17-92, miR-181, miR-29) can regulate the activated phenotype of CLL cells and/or fitness of the surfaceimmunoglobulin (sIg) B cell receptor (BCR) complex expressed by CLL cells, thereby accounting for the differential leukemia-cell expression of these miRNAs in different CLL prognostic subgroups. How these miRNAs influence cellular activation and/or BCR signaling through the post-transcriptional regulation of critical signaling molecules (e.g. Lyn, Syk, BTK, SHIP-1, SHP1) is a topic of current research.
\end{abstract}

\section{Keywords}

CLL; miRNA; ZAP-70; BCR-signalling; prognostic marker; immunoglobulin

\section{Introduction}

The seminal observation that the miR-15a-16-1 cluster located at the $13 \mathrm{q} 14$ region is deleted or deregulated in the leukemia cells of $\sim 50 \%$ of all cases of chronic lymphocytic leukemia (CLL) [1] provided the first evidence that the small non-coding RNAs, known as microRNAs, could contribute to human disease. We now know that there are several hundred functional microRNA genes in the human genome (miRBase v. 19) that can each influence post-transcriptional expression of multiple genes (dozens to hundreds) by inhibiting the translation and/or stability of target messenger RNA (mRNA) [2]. Since the initial observation that miR-15a/16-1 might contribute to CLL pathogenesis, there have been numerous studies examining the potential contribution of these and other microRNAs to the

\section{(C) 2013 Informa UK, Ltd.}

Correspondence: Marek Mraz, MD, PhD/Thomas J. Kipps, MD, PhD, Rebecca and John Moores Cancer Center, 3885 Health Science Dr., La Jolla, CA 9209, USA. Tel: +1-858-5345400. marek.mraz@email.cz/tkipps@ucsd.edu.

Potential conflict of interest: Disclosure forms provided by the authors are available with the full text of this article at www.informahealthcare.com/lal. 
pathogenesis and/or progression of CLL. We reviewed this rapidly accumulating knowledge in this journal in 2009 [3], when the functional consequences of such differences in microRNA expression were largely unclear. This brief review focuses attention on selected microRNAs that are frequently studied in CLL and that appear most likely to contribute to the (dys)regulation of BCR signaling in CLL B cells (Table I).

\section{Role of microRNAs in BCR structure and signaling}

The stage-specific expression of various microRNAs during the maturation of B cells suggests that they play a role in normal B cell development [4]. Consistent with this notion are studies in mice made deficient of the enzyme Dicer, which is required for the production of mature microRNAs. Mice with conditionally deleted Dicer in CD19+ cells develop selfreactive antibodies and autoimmunity associated with increased numbers of marginal-zone B cells and decreased-numbers of follicular B cells [5]. The molecular basis of these observations remains largely unclear, but might relate to recent studies that indicate that $\mathrm{B}$ cell receptor (BCR) signaling and immunoglobulin production can be regulated by microRNAs [2,6]. In turn, BCR stimulation can alter the expression of certain microRNAs, and such BCR-regulated microRNAs might contribute to the orchestration of B cell proliferation, apoptosis and/or sensitivity to BCR signaling [6].

Analysis of the differential expression of microRNAs by CLL cells that use unmutated versus mutated immunoglobulin heavy-chain variable subgenes (IGHV) and/or express versus lack expression of the zeta-associated protein of $70 \mathrm{kDa}$ (ZAP-70) revealed that microRNAs have specific expression profiles in these CLL subtypes, and are also different from normal B cells. Altogether $\sim 18$ microRNAs were identified as differentially expressed between CLL cases divided based on the germline homology of their IGHV and/ or ZAP-70 expression (reviewed in [3]). Some of these differentially expressed microRNAs (such as miR-29, miR-181) were implicated in the regulation of anti-apoptotic proteins myeloid leukemia cell differentiation protein 1 (Mcl1) or T-cell leukemia 1 (Tcl1) [3], but an unbiased approach for identification of genes regulated by these microRNAs is still lacking. Results of these studies, however, suggested that microRNAs might contribute to regulation of the activated phenotype of CLL B cells and/or heterogeneity in BCR signaling propensity observed among CLL cell samples. Following this hypothesis, two recent publications compared the changes in microRNA expression after stimulation of BCR or Toll-like receptors on normal and CLL B cells. Li and colleagues [7] noted that the microRNA expression profile of CLL cells resembles that of B cells activated by treatment with antiimmunoglobulin M (IgM) or CpG. In general, the differences in microRNA expression profile between " germinal center" and " non-germinal center" B cells resemble those observed by expression profiling of protein-coding genes [4]. Additionally, Bomben and colleagues [8] suggested that the microRNAs induced upon treatment with $\mathrm{CpG}$ regulated genes encoding pro-survival and growth-promoting proteins induced by stimulation of Tolllike receptors. This appears in part to be regulated by c-MYC, which can bind the promoters of microRNAs, such as those of the miR-17-92 cluster, to induce expression of such microRNAs. Additionally, certain Toll-like receptors can also be directly stimulated upon binding microRNA molecules secreted by different cell types and/or transported via exosomes. This could be an important link between the tumor microenvironment and the 
intracellular machinery that orchestrates B cell activation and responsiveness to microenvironmental stimuli.

It is noteworthy that many of the microRNAs that are affected by BCR-stimulation in CLL and/or normal B cells are also those that are differentially expressed between cases of CLL with favorable versus unfavorable prognosis (Table I). This suggests that microRNAs might directly or indirectly influence activation in response to BCR ligation and/or disease progression in CLL. However, only a few of the micro-RNAs are known to behave like classical proto-oncogenes, with the possible exception of miR-155, which is expressed at high levels in B cell malignancies and can induce polyclonal B cell expansion and lymphomas in transgenic mice [2]. MicroRNA-155 is up-regulated in response to BCR ligation, and appears to play a role in the development of $\mathrm{T}$ and $\mathrm{B}$ cell immunity, particularly the formation and maintenance of plasma cells secreting IgG [9]. Microarray analysis revealed that deletion of miR-155 may result in the enhanced expression of a large number of target genes [2], most notably the transcription factor PU.1, activation-induced cytidine deaminase (AID) and BCR-associated phosphatase SHIP-1. As is likely the case with all microRNAs, however, the effects of increased or decreased expression of miR-155 are context-dependent and vary depending upon the relative expression of numerous target mRNAs in any given cell type. Recently, we found in CLL cells that PU.1 is likely targeted by miR-155, which is expressed depending upon the methylation status of its promoter and the transcription factor MYB [10]. The induced expression of miR-155 in B cells following BCR ligation requires cellular activation via extracellular signal-regulated kinase (ERK)-, Jun N-terminal kinase (JNK)- and nuclear factor- $\kappa \mathrm{B}(\mathrm{NF}-\kappa \mathrm{B})$-dependent pathways, which are themselves regulated by microRNAs [11,12]. In this regard, it is noteworthy that Epstein - Barr virus (EBV)-encoded latent membrane protein-1 (LMP1) contributes to EBV' $s$ oncogenic potential by up-regulating miR-155 in the infected B cells through activation of $\mathrm{NF}-\kappa \mathrm{B}[12]$.

It is not surprising that the studies of miR-155 provided the first functional evidence for the role of microRNAs in BCR signaling in CLL. It was found that CLL cells with low levels of the miR-155 target SHIP-1 tend to have higher expression of miR-155 and higher capacity for BCR signaling ([13] and manuscript in preparation). In CLL it seems that miR-155mediated regulation of SHIP-1 phosphatase plays an important role in counterbalancing (inhibiting) the BCR signaling capacity. However, the role for other microRNAs in regulation of the complex BCR signaling cascade and BCR-engaged kinases (such as Lyn, Syk, BTK) remains one of the highly interesting, yet poorly explored, questions in the biology of normal and malignant B cells.

The aberrant expression of miR-155 was observed not only in lymphoproliferative disease, but also in many autoimmune conditions, including rheumatoid arthritis, multiple sclerosis and systemic lupus erythematosus, which makes it a potentially interesting therapeutic target. In recently published studies, investigators used anti-sense oligonucleotides specific for miR-155 to inhibit the growth of B cell lymphomas in mice [14]. These studies demonstrated the feasibility of using anti-miR oligonucleotides to inhibit tumor growth in vivo. 
That miR-155 is expressed at relatively high levels in aggressive CLL stands in contrast to what is observed with many other microRNAs in this disease, in which relatively low levels of certain microRNAs are associated with more aggressive disease (Table I). For example, activation via BCR ligation can reduce the levels of miR-29c, miR-150, miR-181b or miR-223, and relatively low-level expression of these microRNAs is found in CLL cells of patients with shorter overall survival and/or time to initial treatment [7,15-17]. Reduced expression of miR-181b, which is associated with increased expression of its notable target genes (e.g. Mcl1 and Bcl2), is a marker of disease progression in CLL [18]. The downregulation of putative tumor-suppressor microRNAs in aggressive cancer subtypes is a frequent phenomenon, and in the case of CLL may lead to overexpression of anti-apoptotic molecules or potentiate BCR signaling. We have found that a large-scale microarray-based approach to the identification of target mRNAs regulated by such down-regulated microRNAs is feasible in CLL, and could help to better uncover the extent of their contribution to BCR pathway (dys)regulation ([17] and manuscript in preparation). The integration of microRNA and gene-expression profiling represents a novel approach that can also be used to identify protein-coding genes with important functions in CLL [17].

It remains unclear to what extent the deregulation of microRNAs represents the cause or the consequence of aberrant BCR signaling or if it contributes, for example, to the maintenance of unbalanced BCR activation in CLL cells, thus favoring malignant B cell clone survival. It is evident that microRNA expression in CLL is influenced by aberrant methylation and acetylation of their promoter regions, which could be due to the response to BCR stimulation as shown previously for protein-coding genes. In these situations, the use of histone deacetylases can induce/restore microRNA expression and sensitize CLL cells to apoptotic stimuli [19], which partially uncovers the mechanism of action for chromatin structure-modifying drugs.

Considering the importance of immunoglobulin gene rearrangement in the development of normal and neoplastic B cells, it is interesting to note that the human Ig locus encodes a microRNA, namely miR-650, which is housed in several light chain variable subgenes of the V2 family. The regulation of miR-650 appears to be coupled with expression of the immunoglobulin lambda light chains, which represents a peculiar mechanism for the regulation of microRNA levels. The expression of miR-650 is associated with CLL prognosis and influences B cell proliferation through regulation of several target genes [20]. Currently, it remains unclear whether any other immunoglobulin genes give rise to noncoding RNAs and how this developed in the evolution of the immunoglobulin locus.

\section{Conclusions}

Recently published studies provide evidence that microRNAs are involved in the BCR pathway and could contribute to the (dys)regulation of BCR signaling in malignant CLL B cells. MicroRNAs frequently associated with CLL biology and the effect of B cell activation/BCR stimulation on their expression are summarized in Table I. These novel observations at least partially explain the differences in microRNA expression between different CLL prognostic subtypes. Conceivably, microRNA inhibitors or mimics could be 
used to manipulate the response to BCR signaling, a prospect that has potential therapeutic implications for patients with B cell malignancies.

\section{Acknowledgments}

Th is work was supported by IGA MZCR NT11218-6/2010 (M.M.), an NIH grant from the CLL Research Consortium (P01-CA081534) to T.J.K. and by the Blood Cancer Research Fund at the UC San Diego Moores Cancer Center.

\section{References}

1. Calin GA, Dumitru CD, Shimizu M, et al. Frequent deletions and down-regulation of micro-RNA genes miR15 and miR16 at 13q14 in chronic lymphocytic leukemia. Proc Natl Acad Sci USA. 2002; 99:15524-15529. [PubMed: 12434020]

2. Xiao C, Rajewsky K. MicroRNA control in the immune system: basic principles. Cell. 2009; 136:26-36. [PubMed: 19135886]

3. Mraz M, Pospisilova S, Malinova K, et al. MicroRNAs in chronic lymphocytic leukemia pathogenesis and disease subtypes. Leuk Lymphoma. 2009; 50:506-509. [PubMed: 19347736]

4. Basso K, Sumazin P, Morozov P, et al. Identification of the human mature B cell miRNome. Immunity. 2009; 30:744-752. [PubMed: 19446474]

5. Belver L, de Yébenes VG, Ramiro AR. MicroRNAs prevent the generation of autoreactive antibodies. Immunity. 2010; 33:713-722. [PubMed: 21093320]

6. Kluiver JL, Chen CZ. MicroRNAs regulate B-cell receptor signaling-induced apoptosis. Genes Immun. 2012; 13:239-244. [PubMed: 22357493]

7. Li S, Moffett HF, Lu J, et al. MicroRNA expression profiling identifies activated B cell status in chronic lymphocytic leukemia cells. PLoS One. 2011; 6:e16956. [PubMed: 21408091]

8. Bomben R, Gobessi S, Bo MD, et al. The miR-17-92 family regulates the response to toll-like receptor 9 triggering of CLL cells with unmutated IGHV genes. Leukemia. 2012; 26:1584-1593. [PubMed: 22343732]

9. Vigorito E, Perks KL, Abreu-Goodger C, et al. microRNA-155 regulates the generation of immunoglobulin class-switched plasma cells. Immunity. 2007; 27:847-859. [PubMed: 18055230]

10. Viargova K, Curik N, Burda P, et al. MYB transcriptionally regulates the miR-155 host gene in chronic lymphocytic leukemia. Blood. 2011; 117:3816-3825. [PubMed: 21296997]

11. Yin Q, Wang X, McBride J, et al. B-cell receptor activation induces BIC/miR-155 expression through a conserved AP-1 element. J Biol Chem. 2008; 283:2654-2662. [PubMed: 18048365]

12. Gatto G, Rossi A, Rossi D, et al. Epstein-Barr virus latent membrane protein 1 trans-activates miR-155 transcription through the NF-kappaB pathway. Nucleic Acids Res. 2008; 36:6608-6619. [PubMed: 18940871]

13. Chen L, Cui B, Chen G, et al. miR-155 contributed to chronic lymphocytic leukemia survival by modulation of BCR-signalling. Blood. 2011; 118(Suppl. 1) Abstract 620.

14. Zhang Y, Roccaro AM, Rombaoa C, et al. LNA-mediated anti-microRNA-155 silencing in lowgrade B cell lymphomas. Blood. 2012; 120:1678-1686. [PubMed: 22797699]

15. Mraz M, Malinova K, Kotaskova J, et al. miR-34a, miR-29c and miR-17-5p are downregulated in CLL patients with TP53 abnormalities. Leukemia. 2009; 23:1159-1163. [PubMed: 19158830]

16. Stamatopoulos B, Meuleman N, Haibe-Kains B, et al. microRNA-29c and microRNA-223 downregulation has in vivo significance in chronic lymphocytic leukemia and improves disease risk stratification. Blood. 2009; 113:5237-5245. [PubMed: 19144983]

17. Mraz M, Rassenti ZL, Ghia EM, et al. The target genes and prognostic significance of miR-150 in chronic lymphocytic leukemia. Blood. 2012; 120(Suppl. 1) Abstract 3859.

18. Visone R, Veronese A, Rassenti LZ, et al. miR-181b is a biomarker of disease progression in chronic lymphocytic leukemia. Blood. 2011; 118:3072-3079. [PubMed: 21636858] 
19. Sampath D, Liu C, Vasan K, et al. Histone deacetylases mediate the silencing of miR-15a, miR-16, and miR-29b in chronic lymphocytic leukemia. Blood. 2012; 119:1162-1172. [PubMed: 22096249]

20. Mraz M, Dolezalova D, Plevova K, et al. MicroRNA-650 expression is influenced by immunoglobulin gene rearrangement and affects the biology of chronic lymphocytic leukemia. Blood. 2012; 119:2110-2113. [PubMed: 22234685] 


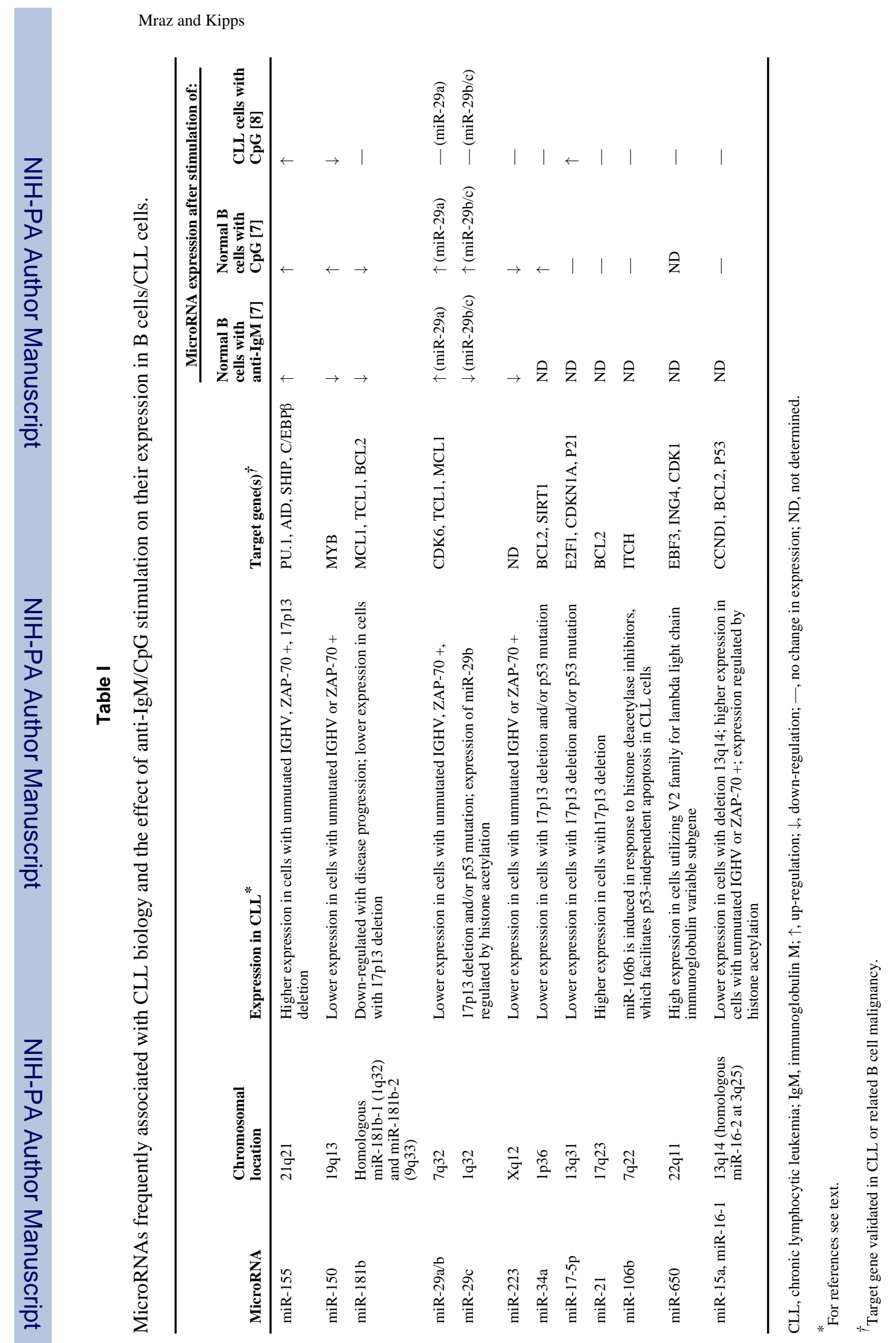

Page 7 